\title{
A speech-based computational auditory signal processing and perception model
}

\author{
Relaño-Iborra, Helia; Zaar, Johannes; Dau, Torsten
}

Published in:

Journal of the Acoustical Society of America

Link to article, DOI:

$10.1121 / 1.5129114$

Publication date:

2019

Document Version

Publisher's PDF, also known as Version of record

Link back to DTU Orbit

Citation (APA):

Relaño-lborra, H., Zaar, J., \& Dau, T. (2019). A speech-based computational auditory signal processing and perception model. Journal of the Acoustical Society of America, 146(5), 3306-3317.

https://doi.org/10.1121/1.5129114

\section{General rights}

Copyright and moral rights for the publications made accessible in the public portal are retained by the authors and/or other copyright owners and it is a condition of accessing publications that users recognise and abide by the legal requirements associated with these rights.

- Users may download and print one copy of any publication from the public portal for the purpose of private study or research.

- You may not further distribute the material or use it for any profit-making activity or commercial gain

- You may freely distribute the URL identifying the publication in the public portal

If you believe that this document breaches copyright please contact us providing details, and we will remove access to the work immediately and investigate your claim. 


\section{A speech-based computational auditory signal processing and perception model}

Helia Relaño-Iborra, Johannes Zaar, and Torsten Dau

Citation: The Journal of the Acoustical Society of America 146, 3306 (2019); doi: 10.1121/1.5129114

View online: https://doi.org/10.1121/1.5129114

View Table of Contents: https://asa.scitation.org/toc/jas/146/5

Published by the Acoustical Society of America

\section{ARTICLES YOU MAY BE INTERESTED IN}

The importance of a broad bandwidth for understanding "glimpsed" speech

The Journal of the Acoustical Society of America 146, 3215 (2019); https://doi.org/10.1121/1.5131651

Corner vowels in males and females ages 4 to 20 years: Fundamental and F1-F4 formant frequencies The Journal of the Acoustical Society of America 146, 3255 (2019); https://doi.org/10.1121/1.5131271

Phonetic change in an Antarctic winter

The Journal of the Acoustical Society of America 146, 3327 (2019); https://doi.org/10.1121/1.5130709

Acoustic and linguistic factors affecting perceptual dissimilarity judgments of voices

The Journal of the Acoustical Society of America 146, 3384 (2019); https://doi.org/10.1121/1.5126697

Bayesian analysis of paired-comparison sound quality ratings

The Journal of the Acoustical Society of America 146, 3174 (2019); https://doi.org/10.1121/1.5131024

Effects of rate and age in processing interaural time and level differences in normal-hearing and bilateral cochlear-implant listeners

The Journal of the Acoustical Society of America 146, 3232 (2019); https://doi.org/10.1121/1.5130384 


\title{
A speech-based computational auditory signal processing and perception model
}

\author{
Helia Relaño-Iborra, ${ }^{a}$ ) Johannes Zaar, and Torsten Dau \\ Hearing Systems Section, Department of Health Technology, Technical University of Denmark, \\ DK-2800 Kgs. Lyngby, Denmark
}

(Received 9 March 2019; revised 29 August 2019; accepted 21 September 2019; published online 13 November 2019)

\begin{abstract}
A new speech intelligibility prediction model is presented which is based on the Computational Auditory Signal Processing and Perception model (CASP) of Jepsen, Ewert, and Dau [(2008). J. Acoust. Soc. Am. 124(1), 422-438]. The model combines a non-linear auditory-inspired preprocessing with a backend based on the cross-correlation between the clean and the degraded speech representations in the modulation envelope domain. Several speech degradation and speech enhancement algorithms were considered to study the ability of the model to predict data from normal-hearing listeners. Degradations of speech intelligibility due to additive noise, phase-jitter distortion, and single-channel noise reduction as well as improved speech intelligibility due to ideal binary mask processing are shown to be successfully accounted for by the model. Furthermore, the model reflects stimulus-level dependent effects of auditory perception, including audibility limitations at low levels and degraded speech intelligibility at high levels. Given its realistic non-linear auditory processing frontend, the speech-based computational auditory signal processing and perception model may provide a valuable computational framework for studying the effects of sensorineural hearing impairment on speech intelligibility. (c) 2019 Acoustical Society of America.
\end{abstract}

https://doi.org/10.1121/1.5129114

[MIM]

Pages: 3306-3317

\section{INTRODUCTION}

Speech perception and communication have been an area of intense research for many years, with contributions from numerous disciplines within psychology, physiology, linguistics, acoustics, signal processing, and others. Among various experimental and theoretical paradigms to study speech perception, and in particular speech intelligibility (SI), computational models have been useful to relate the properties of the sound entering the ear to different measures of speech reception, such as, e.g., phoneme, word, and sentence recognition scores. From the first developments in the early 20th century, with models such as the Articulation index (AI; French and Steinberg, 1947; ANSI, 1969; for an overview see Allen, 1996), to the widely used models of SI developed in the 1980s, such as the Speech Intelligibility Index (SII; Pavlovic, 1987; ANSI, 1997) and the Speech Transmission Index (STI; Steeneken and Houtgast, 1980; IEC, 2003) to recent approaches that make use of heavilytrained automatic speech recognizers (ASRs; e.g., Schädler et al., 2015; Kollmeier et al., 2016), these models have been used to investigate which properties of the sound and which auditory processes dominate people's ability to process speech signals successfully.

While the most powerful SI models succeed in predicting SI across a large range of acoustic conditions in normalhearing $(\mathrm{NH})$ listeners, they typically face a challenge when it comes to characterizing the commonly observed reduction of SI in hearing-impaired (HI) listeners (e.g., Glasberg and

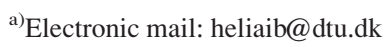

Moore, 1989; Peters et al., 1998). In fact, linking sources of hearing impairment to specific deficits in SI has been a difficult endeavor. HI listeners do not show a homogeneous pattern in their behavior, and while hearing impairment has traditionally been characterized by the audiogram (ISO, 1998), i.e., a measure of sensitivity loss, it has been shown that people with similar audiograms can perform quite differently in speech tasks (Ching et al., 1998; Lorenzi et al., 2006; Strelcyk and Dau, 2009; for a review see LópezPoveda, 2014). Classical modelling approaches, like the AI and the SII, have been adapted to account for hearing impairment, but rely solely on the information provided by the audiogram and have thus only limited applicability (Pavlovic et al., 1986; Payton and Uchanski, 1994; Rhebergen et al., 2010; Meyer and Brand, 2013). On the other hand, sophisticated automatic speech recognition (ASR) based approaches, like the Framework for Auditory Discrimination Experiments (FADE, Schädler et al., 2015; Schädler et al., 2016), while powerful predictors of $\mathrm{NH}$ speech intelligibility, offer only limited insights into the involved auditory processes since the cue extraction from the internal representations of the signals is delegated to a highly trained ASR whose performance relies on the amount and type of (over-)training and less on the actual importance of the selected features for human listeners. Furthermore, such models require explicit individualized fitting of parameters in order to account for HI data (Kollmeier et al., 2016).

Yet another approach would be to make assumptions about the sources of a given hearing impairment and to simulate the effects of such deficits on the internal auditory representation of the signals. For example, Scheidiger et al. (2018) 
proposed a model that employs an auditory nerve (AN) simulation (Carney, 1993; Zilany et al., 2014) followed by a bandpass modulation filter centered at a frequency $(125 \mathrm{~Hz})$ close to the fundamental frequency $\left(F_{0}\right)$ of the target speech. The model was shown to account for speech reception thresholds (SRTs; i.e., the signal level at which $50 \%$ of speech units are correctly understood by the listener) obtained in NH listeners for different interferer types. The model also accounted for the elevated SRTs obtained in HI listeners by adjusting some of the parameters of the frontend processing to the individual listener. However, many of the details in the data of the individual HI listeners could not be captured. Furthermore, within the model, SI was assumed to be proportional to the dissimilarity between the noisy speech and the noise alone, which limits the applicability of the model to listening conditions where noise is present.

In the present study, a conceptually similar approach to the one proposed by Scheidiger et al. (2018) is presented. This approach also utilizes a detailed non-linear auditory model to predict SI but considers clean speech as the reference instead of noise (alone), such that the model is not limited to conditions where noise is present. As an auditory frontend, the preprocessing of the computational auditory signal processing and perception model (CASP; Jepsen et al., 2008) was chosen. CASP has been demonstrated to successfully predict psychoacoustic detection and masking data of NH listeners obtained in basic psychophysical conditions of, e.g., spectral masking, forward masking, intensity discrimination, and amplitude modulation detection and masking. Furthermore, Jepsen and Dau (2011) demonstrated that this model can be adapted to account for data obtained in individual $\mathrm{HI}$ listeners in different psychoacoustic experiments. Here, the original CASP model backend processing, an "optimal detector," was modified towards an SI prediction framework, using a correlation-based backend similar to the ones proposed in the Short-Time Objective Intelligibility measure (STOI; Taal et al., 2011) and the correlation-based speech-based Envelope Power Spectrum Model (sEPSM ${ }^{\text {corr }}$; Relaño-Iborra et al., 2016).

The resulting model, referred to here as the "speechbased" computational auditory signal processing and perception model (sCASP), was validated as a predictor of the intelligibility of Danish sentences measured in NH listeners in conditions of additive noise, phase jitter distortion, spectral subtraction, and ideal binary mask processing (cf. Relaño-Iborra et al., 2016). The reasoning behind selecting these specific conditions was that (i) they cover listening conditions where intelligibility decreases due to degradations in the speech signal as well as conditions where intelligibility increases due to speech enhancement algorithms, and that (ii) the same datasets as for the evaluation of the sEPSM ${ }^{\text {corr }}$ model were used, allowing us to explore the effects of the non-linear processing frontend on the SI predictions by means of a direct comparison.

In addition, the behavior of the model at different stimulus presentation levels was assessed. At low overall stimulus levels, the SRT increases (i.e., SI decreases) and the audibility of the speech signal becomes the only contributor to intelligibility. The corresponding SRT values are higher than those obtained at medium noise levels (Plomp, 1978, 1986). At high overall stimulus levels, the frequency resolution of the auditory system decreases (Glasberg and Moore, 2000) and the amount of upward spread of masking due to the noise increases (Egan and Hake, 1950; Glasberg and Moore, 2000; Summers and Molis, 2004), resulting again in increased SRT values (i.e., reduced SI) as compared to the medium levels ("roll-over"). This reduction in SI has been shown for consonants (Hornsby et al., 2005), words (Pickett and Pollack, 1958; Studebaker et al., 1999) and sentences (e.g., French and Steinberg, 1947; Speaks et al., 1967; Festen, 1993). Due to the common assumption of linearity, most SI models are unable to predict these level effects, unless a dedicated parameter is included for that purpose (e.g., "distortion factor" in SII, ANSI, 1997). Here, it was tested if the leveldependent effects on SI are captured by the sCASP model using an analysis based on the audibility and distortion model of Plomp (1978, 1986). Predictions were compared to data from Summers and Molis (2004) obtained with different interferers presented at high levels in NH listeners and discussed in terms of the model's potential value for predicting consequences of hearing impairment on SI.

\section{MODEL DESCRIPTION}

The proposed sCASP implementation closely follows that of the original CASP model. The model receives the unprocessed clean speech and the noisy or degraded speech mixture as inputs (i.e., it has a priori knowledge of the speech signal). Both inputs are processed through outer- and middle-ear filtering, a non-linear auditory filterbank, envelope extraction, expansion, adaptation loops, a modulation filterbank, and a second-order envelope extraction for modulation channels above $10 \mathrm{~Hz}$. The internal representations produced at the output of these stages are analyzed using a correlation-based backend. Figure 1 shows a flowchart of the main model stages.

\section{A. Auditory preprocessing stages}

The input signals to the model are the clean speech (referred to as the "template" in the following) and the processed/degraded speech (referred to as the "target"). The signals are first filtered through two finite impulse response filters, which replicate the transfer functions of the outerand middle-ear in the human auditory system (Goode et al., 1994; Pralong and Carlile, 1996; Lopez-Poveda and Meddis, 2001). The output of these stages can thus be related to the peak velocity of stapes vibration. Next, the stimulus passes through the dual-resonance non-linear filterbank (DRNL; Lopez-Poveda and Meddis, 2001). The DRNL contains two independent parallel paths. The linear path consists of a linear gain, a cascade of three gammatone filters and a cascade of four lowpass filters. The non-linear path employs a cascade of three gammatone filters and a broken-stick nonlinearity followed by another cascade of four gammatone filters and a cascade of three lowpass filters. The number of cascaded filters and other model parameters are the same as in Jepsen et al. (2008). For more details, further information is provided in Table III of Lopez-Poveda and Meddis (2001) 


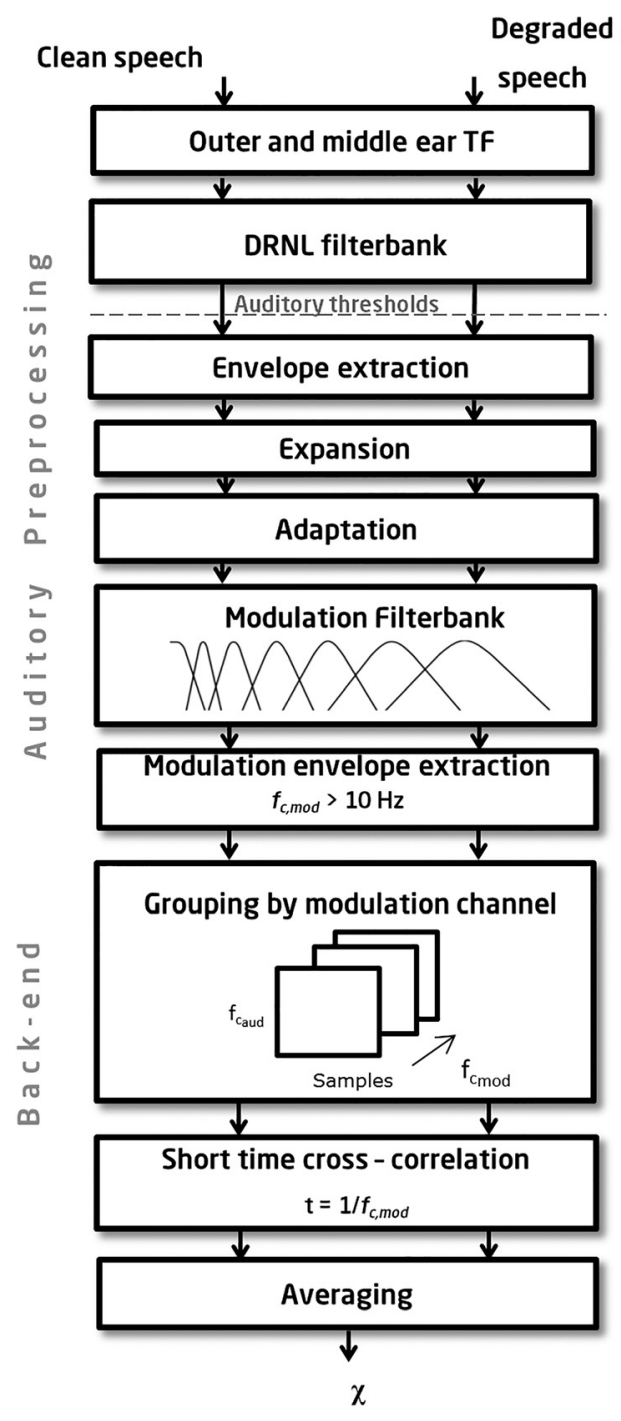

FIG. 1. sCASP processing stages. The input signals are processed though outer- and middle-ear filters, followed by a non-linear filterbank and threshold-simulating noise. The envelope is extracted for each auditory band and quadratically expanded before being passed through a chain of adaptation loops. The signals are then further processed by a modulation filterbank. The resulting internal representations are eventually analyzed using a shortterm correlation for each modulation subband. The resulting correlations are averaged to obtain a unique model output.

and Table I of Jepsen et al. (2008). The time-domain implementation of the DRNL is used, where the output of the model is defined as

$$
y[i]=g \cdot x(i)+\operatorname{sign}(x[i]) \cdot \min \left(a \cdot|x[i]|, b \cdot|x[i]|^{c}\right),
$$

where $i$ refers to the $i$ th sample of the time signal and $x$ and $y$ are the input and output signals, respectively. The first component of the output signal represents the linear gain, and the second component represents the broken stick nonlinearity, which comprises a linear section $(a \cdot|x[i]|)$ and a compressive section $\left(b \cdot|x[i]|^{c}\right)$, with $c$ representing the amount of compression and $a$ and $b$ determining the location of the knee point of the broken-stick nonlinearity. The summed signal of the two paths includes the effects of the non-linear basilar-membrane processing, which accounts for level-dependent compression and auditory-filter tuning.
After this stage, internal noise is added to the outputs of the DRNL model to account for audibility limitations (see details in Sec. II C). The auditory filtering is followed by an envelope extraction stage, implemented as half-wave rectification followed by second-order low-pass filtering ( $f_{\text {cut-off }}$ $=1 \mathrm{kHz}$ ). The resulting envelopes are expanded quadratically into an intensity-like representation.

Effects of adaptation are modelled using a chain of five feedback loops (Püschel, 1988; Dau et al., 1996). The time constants defining the behavior of the adaptation chain are those proposed by Dau et al. (1996). The final stage of the auditory preprocessing block is a modulation analysis, carried out by a low-pass filter $\left(f_{\text {cut-off }}=150 \mathrm{~Hz}\right)$ followed by a bank of frequency-shifted first-order low-pass filters (i.e., they act as band-pass filters) parallel to a second-order lowpass filter $\left(f_{\text {cut-off }}=2.5 \mathrm{~Hz}\right)$. The first two band-pass filters have a constant bandwidth of $5 \mathrm{~Hz}$, while the remaining filter bandwidths are logarithmically scaled with a quality factor $(Q)$ of 2. In order to account for reduced modulation-phase sensitivity (Langner and Schreiner, 1988; Dau, 1996; Dau et al., 1997a,b) for filters above $10 \mathrm{~Hz}$, only the absolute values of the modulation filter outputs are considered ("secondorder envelopes"). For modulation filters centered below $10 \mathrm{~Hz}$, the real part is considered. Additionally, only those modulation subbands whose center frequency $\left(f_{c}\right)$ is below $1 / 4$ of the auditory-band $f_{c}$ are further processed (Verhey et al., 1999) to extract the internal representations of both the template and the target signals.

\section{B. Decision backend processing}

The internal representations of the pre-processed signals contain the dimensions time, audio frequency, and modulation frequency. Recent studies showed that the relative differences in the internal representation across the different auditory channels provide critical information related to the intelligibility of speech signals (e.g., Elhilali et al., 2003; Chabot-Leclerc et al., 2014; Carney, 2018; Scheidiger et al., 2018). Thus, the sCASP model's backend was designed such that it assumes independent processing across modulation channels but analyzes the contribution of all auditory channels simultaneously for each of the modulation frequencies. This is achieved by grouping subbands with the same modulation $f_{c}$ obtained from the processing in each auditory band, effectively generating separate spectrogram-like representations for each modulation channel (referred to as "modulation-spectrograms" in the following). This is in contrast to the sEPSM ${ }^{\text {corr }}$ model, where the three dimensions of the internal representation were considered independently, i.e., the time analysis was performed individually for each audio-frequency and modulation-frequency channel, assuming band independence both in the audiofrequency and the modulation-frequency domain.

Figure 2 shows examples of the sCASP modulationspectrograms for the lowest modulation channel $[2.5 \mathrm{~Hz}$; Figs. 2(c) and 2(d)] and the highest modulation channel [992 Hz; Figs. 2(f) and 2(g)]. They were obtained for a clean sentence at a sound pressure level (SPL) of $65 \mathrm{~dB}$ [waveform shown in Fig. 2(a)] and for the same sentence mixed with speech shaped noise (SSN) at a SNR of $0 \mathrm{~dB}$ [shown in Fig. 


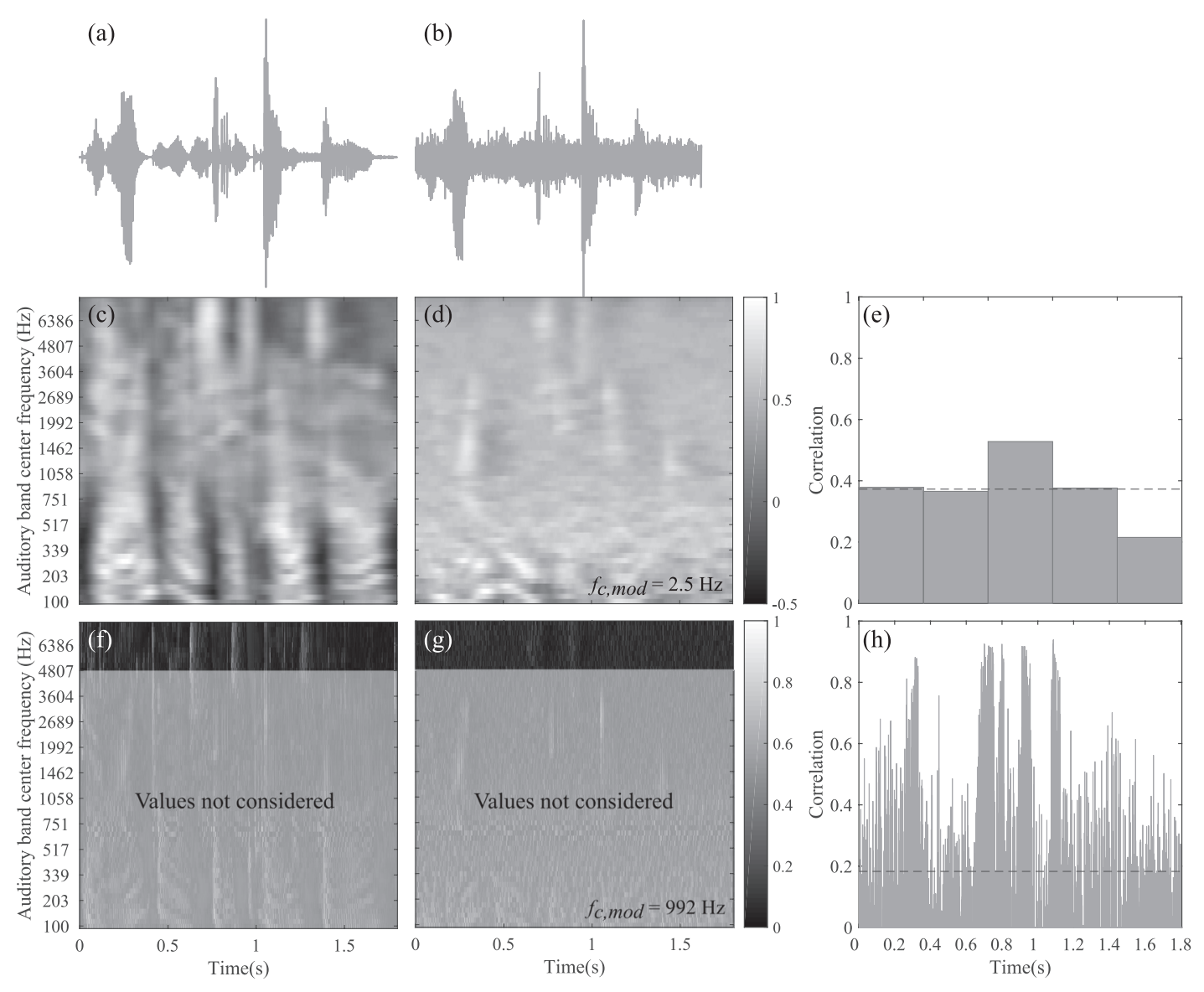

FIG. 2. Panels (a) and (b) show the inputs to the model for an example CLUE sentence ("I regnbuen ses alle farver"/“In the rainbow all colors are seen"), where the template (a) is the clean sentence presented at $65 \mathrm{~dB}$ SPL, and the target (b) is the same sentence mixed with SSN at a $0 \mathrm{~dB}$ SNR. Panels (c) and (d) show the modulation spectrograms for the first modulation band (low-pass filter with $f_{\text {cut-off }}=2.5 \mathrm{~Hz}$ ) for the template and target, respectively. Similarly, panels (f) and (g) show the modulation spectrograms for the highest modulation band (band-pass filter with $f_{c}=992 \mathrm{~Hz}$ ). Note that, within the model, lower modulation frequency bands are processed in terms of their real part and higher modulation frequencies are processed in terms of their absolute value, representing a second-order envelope. The number of auditory channels used to generate the modulation spectrograms depends on the modulation frequency. The rejected values are indicated by the shaded area of panels (f) and (g). For illustration purposes, internal noise was not included and the amplitudes were normalized. Panels (e) and (h) show the correlation values resulting from each target and template pair of spectrograms as a function of time. Each bin represents a time window, whereas the dashed line represents the average correlation value obtained for that modulation frequency.

2(b)]. Due to the selection of modulation channels that are below $1 / 4$ th of the auditory channels' $\mathrm{CF}$, the number of values along the audio frequency axis considered is different across modulation-frequency spectrograms. The rejected values are shaded out in Figs. 2(f) and 2(g). In the model, the modulation-spectrograms of the "template" and the "target" signals are cross-correlated at zero-lag using short time windows with a duration defined for each modulationspectrogram by the inverse of the modulation frequency, as in the sEPSM ${ }^{\text {corr }}$ and mr-sEPSM models (Jørgensen et al., 2013; Relaño-Iborra et al., 2016). Thus, the temporal resolution of the analysis changes across modulation spectrograms, as shown in Figs. 2(e) and 2(h). For each analysis window, the cross-correlation is performed simultaneously across the two dimensions (time and auditory frequency) by collapsing all auditory channels into one single vector. The correlation values are then averaged across time windows and across all modulation frequencies, resulting in a single value at the model output for each pair of input signals. A detailed derivation of the backend processing is provided in the Appendix.

The proposed backend is thus sensitive to acrossfrequency properties in the sound. By correlating the modulation-spectrograms of the target and template, the model captures changes introduced in the target signal both in the time and in the frequency domain. This backend differs from the approach used in the sEPSM ${ }^{\text {corr }}$ in that (i) it does not assume independence across auditory filters but instead computes the joint correlation across time and audio frequency and (ii) it does not require the summation of correlation values across time windows [see Relaño-Iborra et al., 2016, Eq. (3)] but instead averages the correlation values across time (see also Sec. V A). The backend of sCASP also differs from the ideal-observer (IO) backend used in the original CASP model, where no short-time analysis is included and decisions are based on the correlation between the internal representations of masker-plus-suprathreshold signal minus masker alone ("template") and the internal representation of the actual signal mixture minus masker alone ("target"; Dau et al., 1996).

\section{Transformation to SI scores}

The correlation-based output of the proposed model is monotonically related to the SNR of the target stimuli. In 
order to transform the correlation values into speech intelligibility scores, a mapping function is applied. This was done once per speech corpus and it was assumed that it reflects the differences in intelligibility across speech corpora (e.g., syntactic complexity, contextual information, vocabulary size, etc.). The mapping was performed by applying a logistic function to the model output, $\chi$,

$$
\Phi(\chi)=\frac{100}{1+e^{(a \cdot \chi+b)}},
$$

where $a$ and $b$ are the free parameters defined in the fitting condition using a least squares optimization.

This approach is different from the original CASP model where the model was used within the framework of an Ideal Observer (IO) and the stimuli were presented to the model in terms of an alternative-forced choice procedure, i.e., in the same manner as they would be presented to the listener (Dau et al., 1996; Jepsen et al., 2008). In the original CASP model (Jepsen et al., 2008), as in previous IO-based models (Dau et al., 1996; Dau et al., 1997a,b), the tuning of the ideal observer included changes in sensitivity that would otherwise be achieved by introducing internal noise in the model processing chain (i.e., the uncertainty is moved from the actual internal representations to the IO). Thus, when removing the IO stage in SCASP, the model's sensitivity limitation that had been partly realized by the IO internal noise needed to be restored in the preprocessing (for more details about the relationship between internal noise and ideal observer, see the Appendix in Dau et al., 1996). In the sCASP model, the internal noise was implemented as white Gaussian noise, generated independently for each audiofrequency channel and added to the outputs of the DRNL filter before the envelope extraction carried out in the IHC transduction stage. Thus, the internal noise can be considered in the model as representing spontaneous activity occurring at the synapse between the IHCs and the auditory nerve fibers, resulting in spontaneous firing of the auditory nerve fibers. The noise level was adjusted individually for each band in order to account for the $\mathrm{NH}$ hearing thresholds (ISO, 2005), by means of an adaptive procedure tracking the amount of internal noise necessary for a $70.7 \%$ tone-in-quiet detection probability. The adjustment was performed once and kept constant across all simulations.

\section{METHODS}

In order to assess the proposed model's ability to account for speech intelligibility (SI) in human listeners, the model was tested in the same listening conditions as in the study by Relaño-Iborra et al. (2016). This allowed for a direct comparison with the SEPSM $^{\text {corr }}$ model and thus made it possible to evaluate the effects of the detailed auditory preprocessing as well as the back-end processing of sCASP on the predicted SI. The experimental design is briefly described below.

\section{A. Speech materials and model fitting}

The model was validated in four different listening conditions. Two of them considered speech degradations where

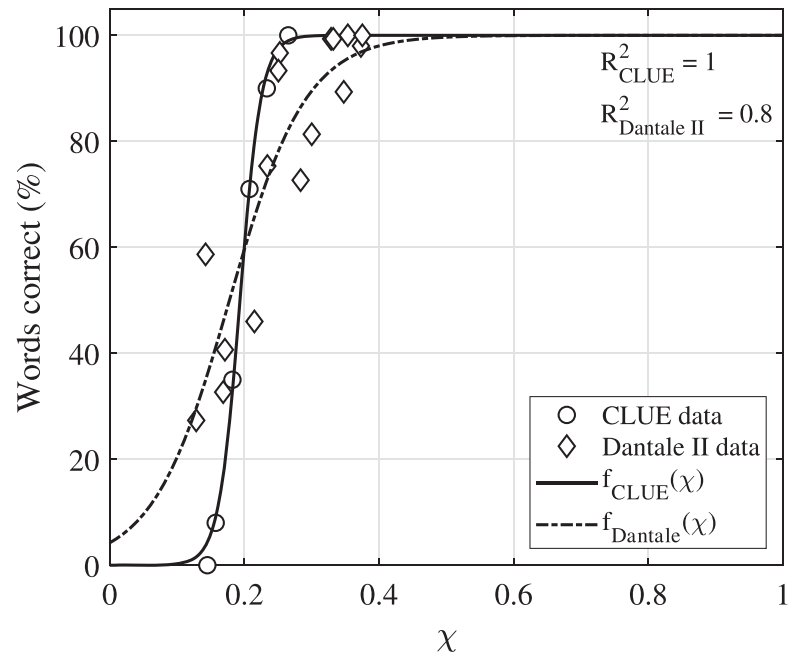

FIG. 3. Fitting curves for the CLUE (solid line) and Dantale II (dashed line) corpora. The data points represent the relationship of human data (ordinate) and model output (abscissa). The circles represent data from the study of Nielsen and Dau (2009) for the condition of CLUE speech mixed with SSN. The diamonds represent the data from the study of Kjems et al. (2009) for the condition of Dantale II sentences mixed with SSN for different IBM configurations.

intelligibility is expected to suffer, namely, speech in the presence of different types of additive interferers as well as distorted, phase-jittered, speech. The two other conditions considered noisy speech enhancement approaches, namely, spectral subtraction and ideal binary mask (IBM) processing. In the latter condition, the Dantale II corpus (Wagener et al., 2003) was used. In all other conditions, the CLUE corpus (Nielsen and Dau, 2009) was used. As mentioned in Sec. II C, a fitting condition is required to transform the model outputs into intelligibility scores using Eq. (2). The model was thus calibrated twice in the present study, once per speech material, using SSN at different SNRs. Thus, the mapping accounts for the intelligibility of the speech material but implies no a priori knowledge of the considered degradations other than the degradation induced by the SSN. For the Dantale II material, all data points corresponding to the SSN condition were utilized for fitting, due to a lack of enough unprocessed SSN data points to obtain a strong fit. Figure 3 shows the fitted functions for the two speech materials. The assigned values of the free parameters are indicated in Table I.

\section{B. Experimental conditions}

First, the effects of additive noise on SI were considered. Three different maskers were employed: (i) speech-shaped noise ("SSN"), (ii) an 8-Hz sinusoidally amplitude-modulated SSN with a modulation depth of one ("SAM"), and (iii) the

TABLE I. Fitting parameters of Eq. (2) used to map model outputs to intelligibility scores for the two different speech corpora.

\begin{tabular}{lrr}
\hline \hline & CLUE & Dantale II \\
\hline$a$ & -63.8 & -17.5 \\
$b$ & 12.3 & 3.1 \\
\hline \hline
\end{tabular}


international speech test signal ("ISTS"; Holube et al., 2010), which is a speech-like interferer, but largely unintelligible. The speech in both the clean template and the mixture target were CLUE sentences with a fixed level of $65 \mathrm{~dB}$ SPL and the noise level in the mixture was adjusted to evaluate SNRs ranging from -27 to $3 \mathrm{~dB}$, with a step size of $3 \mathrm{~dB}$. Predicted SRTs were compared to measured SRTs for the same stimuli as in Jørgensen et al. (2013).

Next, the effects of random phase changes in the speech signal were investigated. The phase jitter was applied to CLUE sentences with a level of $65 \mathrm{~dB}$ SPL mixed with SSN at a fixed SNR of $5 \mathrm{~dB}$. The jitter was applied individually to each time sample of the mixture following:

$$
r(t)=\operatorname{Re}\left\{s(t) e^{j \theta(t)}\right\}=s(t) \cos (\theta(t)),
$$

where $s(t)$ represents the non-processed mixture, $r(t)$ the resulting jittered stimulus, and $\theta(t)$ denotes a random process with a uniform probability distribution between $[0,2 \alpha \pi]$, with $\alpha$ ranging between 0 and 1 (Elhilali et al., 2003). As in the reference human data collected by Chabot-Leclerc et al. (2014), the effect of changes in the $\alpha$ value, i.e., the amount of phase jitter, was investigated in the model framework. Values of $\alpha=0$, $0.125,0.25,0.375,0.5,0.625,0.75,0.875$, and 1 were used.

The model's ability to capture the effects of speech enhancement algorithms was also explored. Two different noise-reduction schemes were considered. Spectral subtraction processing (Berouti et al., 1979) was applied to the noisy speech (consisting of CLUE sentences and SSN) as follows:

$$
\widehat{S(f)}=\sqrt{P_{Y}(f)-\kappa \widehat{P}_{N}(f)}
$$

where $\widehat{\mathrm{S}(\mathrm{f})}$ is the enhanced magnitude spectrum of the noisy mixture after spectral subtraction, $\mathrm{P}_{\mathrm{Y}}(\mathrm{f})$ is the averaged power spectrum of the noisy mixture, and $\widehat{P}_{\mathrm{N}}(\mathrm{f})$ is the averaged power spectrum of the noise alone. The speech level was fixed at $65 \mathrm{~dB}$ SPL and SNRs from -9 to $9 \mathrm{~dB}$ in $3 \mathrm{~dB}$ steps were considered. Values for the over-subtraction factor, $\kappa$, of $0,0.5$, $1,2,4$, and 8 were used. The simulations were compared with data published in Jørgensen and Dau (2011).

Finally, the model was evaluated on speech enhanced with IBM. For that purpose, the dataset from Kjems et al. (2009) was considered. It consists of intelligibility scores for two SNR mixtures of Dantale II sentences (corresponding to $50 \%$ and $20 \%$ intelligibility) with four different interferers: SSN, carcabin noise ("Car"), noise produced by bottles on a conveyor belt ("Bottle"), and two people speaking in a cafeteria ("Café"). Each combination of noise masker and SNR was processed with several IBM configurations, as follows:

$$
\operatorname{IBM}(t, f)= \begin{cases}1 & \text { if } \operatorname{SNR}(t, f)>\mathrm{LC} \\ 0 & \text { otherwise }\end{cases}
$$

where LC corresponds to the local criterion, defined as the SNR threshold at which the $(t, f)$ bin is assigned 1 or 0 in the mask. Eight different values of LC were considered for each interferer. In total, 64 data-points were considered
(8 LC $\times 2 \mathrm{SNR} \times 4$ interferers). To simplify the comparison across the different SNR mixtures, the relative criterion defined as RC $=$ LC-SNR is used here.

\section{Assessment of stimulus-level effects in SI}

In all experimental conditions described above, the stimulus levels were well above hearing threshold. However, any attempt to use sCASP to predict hearing-loss induced changes in SI requires that the limitations of the $\mathrm{NH}$ auditory system itself can be correctly captured by the model. This was studied in terms of the model's ability to reflect the nonlinear relationship between SI and stimulus level. At low levels, audibility limitations should come into play, whereas at high levels, loss of frequency resolution and increased upward spread of masking should limit the model's performance. One way of characterizing how SI is affected by the overall presentation level of the stimuli is the wellestablished audibility-distortion ${ }^{1}$ model of Plomp (1978, 1986). This model defines the speech level at SRT $\left(L_{S}\right)$ as

$$
L_{S}=10 \log \left[10^{L_{0} / 10}+10^{\left(L_{N}+\Delta L_{S N}\right) / 10}\right],
$$

where $L_{0}$ is the SRT in quiet, $L_{N}$ is the sound pressure level of the noise in $\mathrm{dB}$, and $\Delta L_{S N}$ is the SNR at threshold (i.e., the SRT). This model illustrates that for low noise levels, when the "external" noise is below audibility threshold, the ability to understand speech is limited by the "internal" noise in the auditory system. Here, the Plomp model was considered as a reference to evaluate sCASP at different stimulus levels. Corresponding simulations were carried out where the noise level was fixed at values from -20 to $100 \mathrm{~dB}$ SPL in $10 \mathrm{~dB}$ steps, and the speech level was varied at SNRs ranging from -30 to $+39 \mathrm{~dB}$ in $3 \mathrm{~dB}$ steps. For each fixed noise level, an SRT was obtained and the corresponding $L_{s}$ values were calculated. The Plomp model assumes that, once the noise level is above audibility thresholds, the SRT remains constant with increasing stimulation level. Thus, it does not reflect the reduced SI observed at high levels ("roll-over") in the data of NH listeners (e.g., Pickett and Pollack, 1958; van Wijngaarden et al., 1999; Summers and Molis, 2004; Summers and Cord, 2007). To investigate the model's behavior at high levels, model predictions were compared to SRTs measured by Summers and Molis (2004) at target speech levels of 60, 75, and $90 \mathrm{~dB}$ SPL.

\section{RESULTS}

Figures 4-7 show the results obtained for the different degraded and processed speech conditions. The human data are shown as open squares, while sCASP predictions are depicted using black circles. For comparison, predictions obtained with the sEPSM ${ }^{\text {corr }}$ are shown using gray diamonds. The accuracy of the model's predictions in the suprathreshold conditions is provided in terms of their Pearson's correlation $(\rho)$ with the human data and the mean absolute error (MAE) in Table II. Figures 8 and 9 show the simulations obtained in the experiments with varying levels of the speech and noise stimuli. 


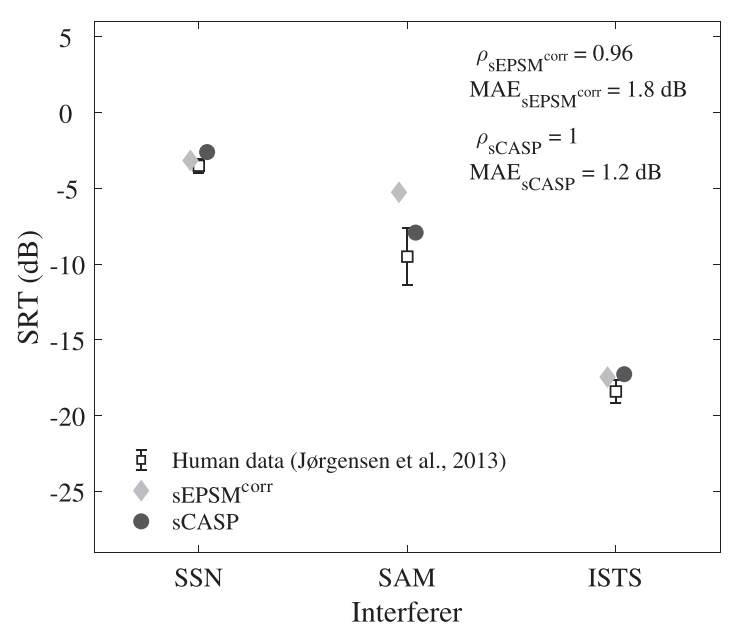

FIG. 4. SRTs for three different noise maskers: SSN, SAM, ISTS. The squares represent the human data from Jørgensen et al. (2013), the gray diamonds represent predictions obtained with the $\mathrm{SEPSM}^{\text {corr }}$ model and the black circles show predictions obtained with the proposed sCASP.

\section{A. Stationary and non-stationary interferers}

Figure 4 shows the measured and simulated SRTs for speech in the presence of several noise maskers. In this experiment, the human data showed reduced SRTs (i.e., greater SI) when the speech was presented in fluctuating maskers as compared to conditions where speech was presented in stationary noise. This phenomenon, known as masking release, can be accounted for by the proposed model $(\rho=1$, MAE $=1.2 \mathrm{~dB})$, slightly improving the results of $\operatorname{sEPSM}^{\text {corr }}(\rho=0.96$, MAE $=1.9 \mathrm{~dB})$. The improvement in performance is due to improved prediction of the SRT measured for the "SAM" interferer, where the non-linear model clearly outperforms the EPSM-based frontend, whereas the predictions of the "ISTS" SRT are very similar for both models.

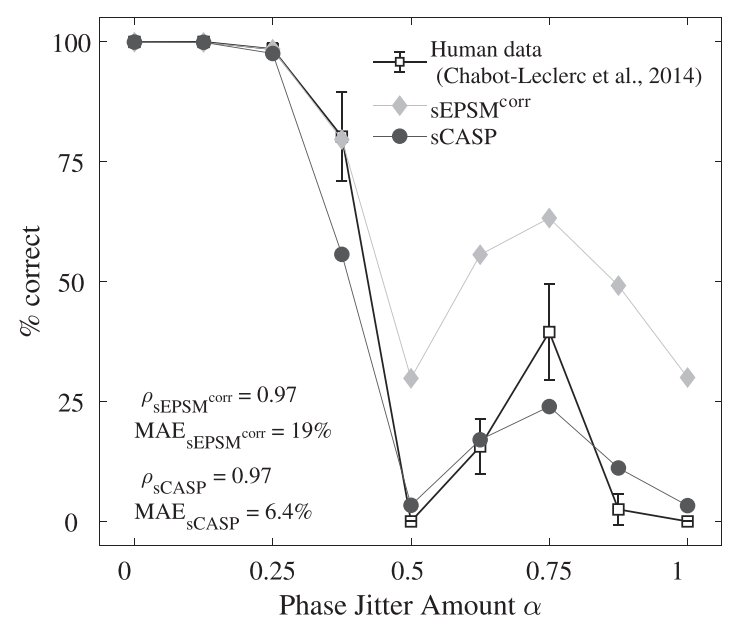

FIG. 5. Intelligibility scores for speech plus SSN, jittered with different amounts of phase jitter. The squares represent human data gathered by Chabot-Leclerc et al. (2014); the gray diamonds represent predictions obtained with the SEPSM $^{\text {corr }}$ model; the black circles show predictions obtained with the proposed sCASP.

\section{B. Phase-jitter distortion}

Figure 5 shows how SI scores change with different amounts of phase jitter distortion. The data, taken from Chabot-Leclerc et al. (2014), show a very distinct nonmonotonic relationship between SI and phase jitter $(\alpha)$, which can be accounted for by the sCASP simulations. The correlation between the sCASP predictions and the data amounts to $\rho=0.97$, the same value as was obtained with the SEPSM $^{\text {corr }}$. However, the MAE of the sCASP simulations is lower $\left(\mathrm{MAE}=6.4 \%\right.$ ) than that of the $\mathrm{sEPSM}^{\text {corr }}$ simulations $(\mathrm{MAE}=19 \%)$. The reason for the improved predictions with sCASP is that the model is able to account for the data also in those conditions where SI fully drops to zero ( $\alpha=0.5$ and $\alpha=1$ ), whereas sEPSM ${ }^{\text {corr }}$, while capturing the main trends in the data, could not account for the low intelligibility scores $(<25 \%)$ induced by the phase-jitter algorithm.

\section{Noise-reduction processing}

The potential of the model as a predictor of SI for enhanced speech was explored. Figure 6 shows the human data (open symbols) and the model predictions for spectrally subtracted speech. Figure 7 shows data and model predictions for speech processed with several IBM configurations. The data show that while the IBM approach generally increases SI in human listeners (Kjems et al., 2009), this is not the case for speech processed by spectral subtraction, for which higher SRTs (i.e., worse SI) were reported with increasing amount of spectral subtraction, $\kappa$ (Jørgensen and Dau, 2011).

The predictive power of $\operatorname{sCASP}(\rho=0.6, \mathrm{MAE}$ $=1.4 \mathrm{~dB})$ was lower than that of the $\operatorname{sEPSM}^{\text {corr }}(\rho=0.82$, $\mathrm{MAE}=0.6 \mathrm{~dB})$ in the spectral subtraction conditions, whereas it was higher in the IBM conditions where it outperforms sEPSM $^{\text {corr }}$ (sCASP: $\rho=0.88$, MAE $=10.0 \% \mathrm{~dB}$; sEPSM $^{\text {corr }}: \rho=0.79$, MAE $=12.2 \%$ ). The reason for the lower accuracy observed in the spectral subtraction conditions is that the model cannot capture the increase in SRT for $\kappa=0.5$ relative to the unprocessed condition $(\kappa=0)$, even though it otherwise correctly describes the trend of increasing SRTs

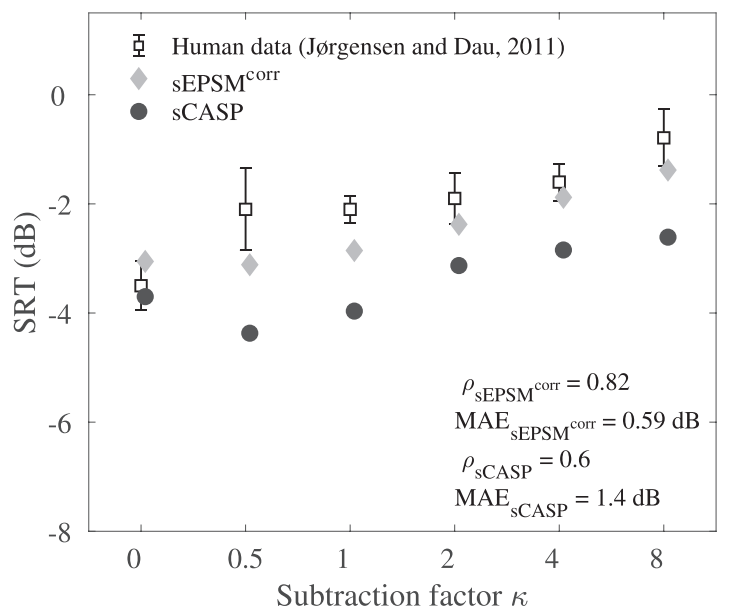

FIG. 6. Human data and model simulations for noisy speech enhanced using different amounts of spectral subtraction, with human data obtained from Jørgensen and Dau (2011) represented in squares. The gray diamonds represent predictions using the SEPSM $^{\text {corr }}$ model and the black circles show predictions using the proposed sCASP model. 


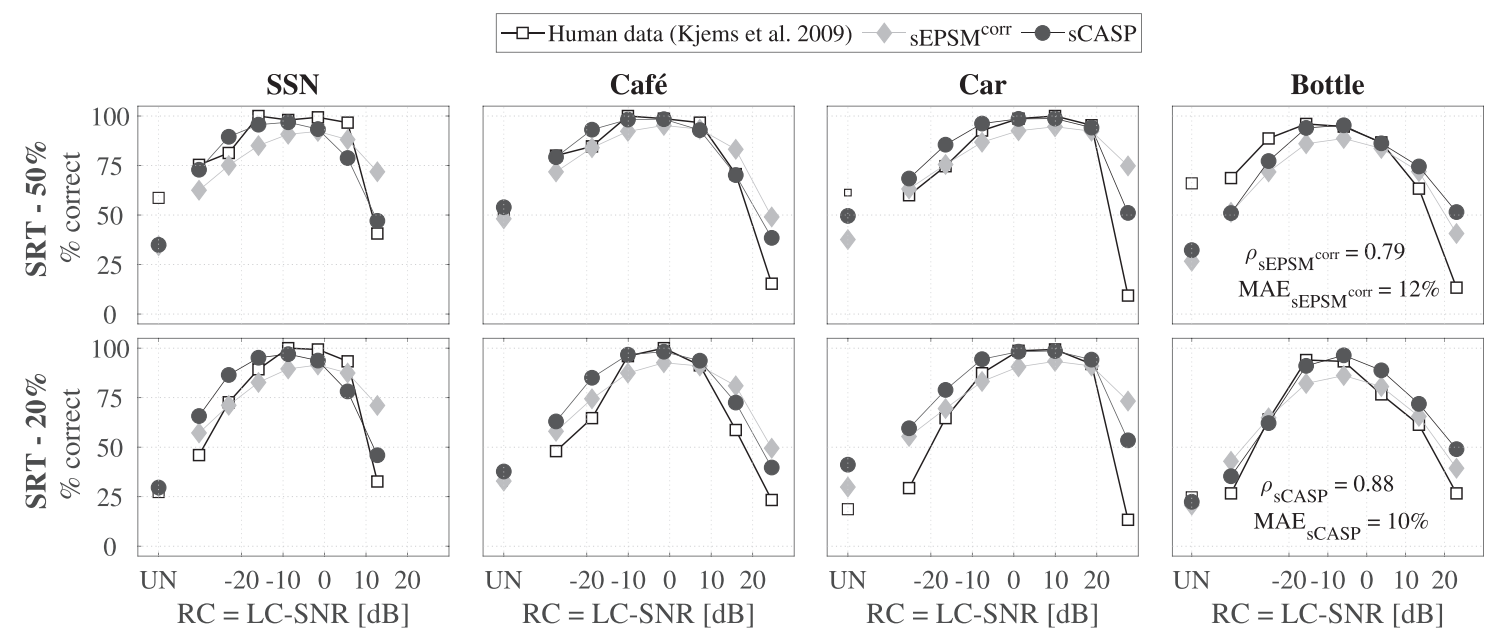

FIG. 7. Speech intelligibility scores for different IBM configurations for two SNR mixtures (rows) and four different interferers (columns), where the squares represent human data from Kjems et al. (2009). The gray diamonds represent predictions using the sEPSM $^{\text {corr }}$ model and the black circles show predictions using the proposed sCASP model.

(i.e., decreasing SI) with increasing spectral subtraction factor. The limitations of the sCASP model in this listening condition are discussed in more detail further below.

\section{Stimulation-level effects}

The dashed line shown in Fig. 8 represents the "idealized" Plomp function for NH listeners in the case of CLUE sentences mixed with SSN. To obtain this function, Eq. (6) from Sec. IIIC was used with $\Delta L_{S N}$ set to $-3.5 \mathrm{~dB}$ (Jørgensen et al., 2013) and $L_{0}$ set to $24 \mathrm{~dB}$ (Nilsson et al., 1994; Nielsen and Dau, 2009). The Plomp function shows two distinct regions: one region $(>25 \mathrm{~dB}$ noise level) where the speech level at SRT increases linearly with the noise level (implying a constant SNR at the SRT, since $\left.S R T=\Delta L_{S N}=L_{S}-L_{N}\right)$ and one region $(<25 \mathrm{~dB}$ noise level $)$, where the speech level at SRT remains constant (implying that the SRT increases with decreasing noise level).

The solid black line in Fig. 8 represents the simulated results for the SSN masker using the sCASP. The simulations accurately follow the Plomp function, with slight deviations towards higher speech levels (i.e., elevated SRTs) at the highest noise levels. For comparison, simulations were also run using the sEPSM ${ }^{\text {corr }}$. In contrast to the simulations obtained with sCASP, the simulated function obtained with $\mathrm{SEPSM}^{\text {corr }}$ (gray line) produces values that are substantially lower than those provided by the Plomp model, indicating that the sEPSM $^{\text {corr }}$ cannot realistically capture audibility limitations.

TABLE II. Accuracy metrics for the two discussed models. $\rho$ indicates the Pearson's correlation with the human data and MAE the mean absolute error.

\begin{tabular}{lccccc}
\hline \hline & \multicolumn{2}{c}{ sCASP } & & \multicolumn{2}{c}{ sEPSM $^{\text {corr }}$} \\
\cline { 2 - 3 } \cline { 5 - 6 } & $\rho$ & MAE & & $\rho$ & MAE \\
\hline Additive noise & 1 & $1.2 \mathrm{~dB}$ & & 0.96 & $1.9 \mathrm{~dB}$ \\
Phase Jitter & 0.97 & $6.4 \%$ & & 0.97 & $19.0 \%$ \\
Spectral Subtraction & 0.60 & $1.4 \mathrm{~dB}$ & & 0.82 & $0.6 \mathrm{~dB}$ \\
IBM processing & 0.88 & $10.0 \%$ & & 0.79 & $12.2 \%$ \\
\hline \hline
\end{tabular}

To further study the effects at high presentation levels, Fig. 9 replots the simulations from Fig. 8, now representing the SRT as a function of the speech level instead of showing the speech level at the SRT. The results for the sCASP model are shown in the middle panel with the solid line representing the SSN masker condition. The corresponding simulations for the $\mathrm{sEPSM}^{\mathrm{corr}}$ are shown in the right panel. Additional simulations for SAM and ISTS interferers were carried out to explore the influence of the masker properties on SI as a function of the signal level. For comparison, the left panel of Fig. 9 shows the data from Summers and Molis (2004) that were obtained in NH listeners using speech mixed with SSN (squares) and a single-talker interferer ${ }^{2}$ (diamonds). It can be seen that the measured SRTs increase with increasing target speech levels, whereby the effect was found to be stronger in the case of the interfering talker.

The sCASP predictions show higher SRTs at higher speech levels than at medium levels for all considered maskers. The

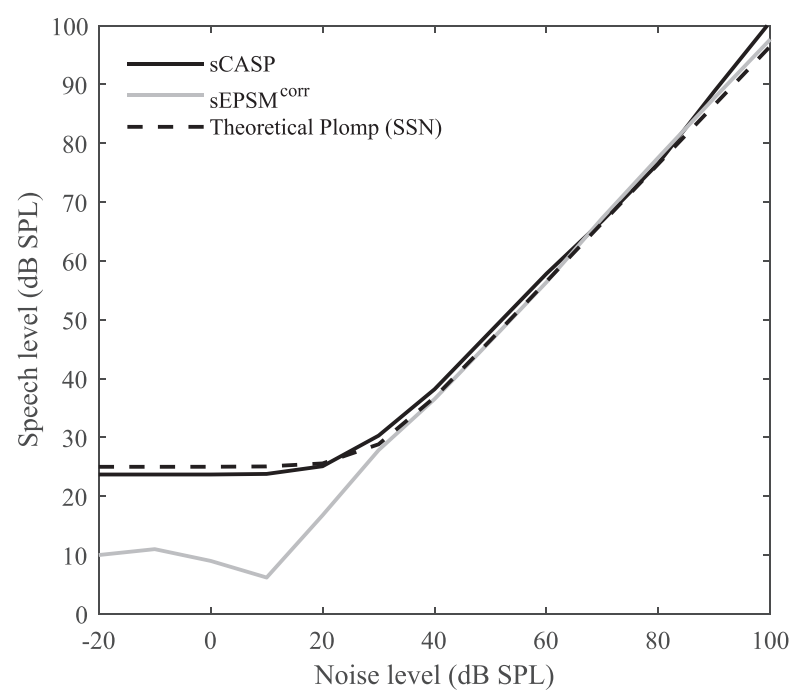

FIG. 8. Values for speech and noise levels at the SRT for different interferers and two different models, sCASP (black line) and sEPSM ${ }^{\text {corr }}$ (gray line). The dashed line represents the ideal Plomp curve for the CLUE speech material in the presence of SSN. 

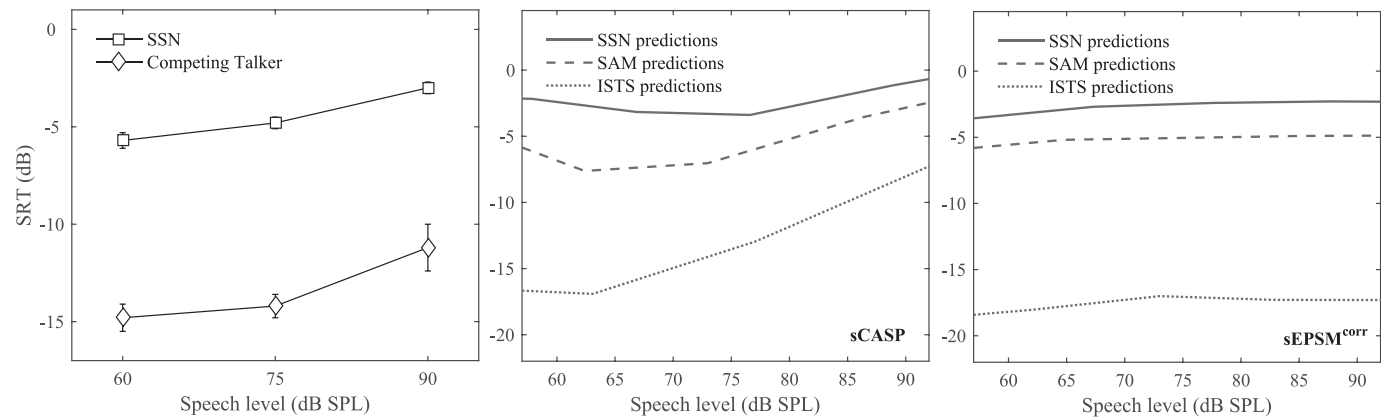

FIG. 9. The left panel shows, as a reference, human data from Summers and Molis (2004), with squares representing SRT data for speech with an SSN masker and open diamonds representing SRTs obtained for speech in the presence of a speech masker. The middle panel shows predictions obtained with the proposed sCASP model, and the right panel shows predictions obtained with the sEPSM ${ }^{\text {corr }}$. Note that the model simulations and human data cannot directly be compared, as the listening material and interferers used differ between the two. Human SRTs are only provided here as a reference of expected trends of SI with level.

increase in SRT is more prominent for the fluctuating maskers than for the stationary masker. Both aspects are consistent with the trends in the data. Furthermore, the sCASP predictions and the data show that the SRT differences obtained between the conditions with modulated vs unmodulated maskers tend to decrease at high stimulation levels. In contrast, the sEPSM ${ }^{\text {corr }}$ does not predict any roll-over effect, nor does the difference between the interferers change with level. Thus, the sEPSM ${ }^{\text {corr }}$ shows the same amount of listening advantage due to fluctuations at high levels and at medium levels, in contrast to the human data (Festen, 1993; Summers and Molis, 2004).

\section{DISCUSSION}

\section{A. Essential model components for SI prediction}

The speech model presented here, characterized by the combination of a non-linear auditory preprocessing with a two-dimensional (2D) short-time correlation analysis of the modulation subbands, accounts for SI in listening conditions where various alternative models have been shown to fail. In the following, it is discussed which processing stages are essential for the accurate predictions obtained in the framework of the proposed model.

Many SI models have faced challenges to account for the release from masking experienced by $\mathrm{NH}$ listeners when listening to speech in the presence of fluctuating maskers. The sCASP model was shown to account for these conditions (Sec. IV A), outperforming the results reported for the sEPSM $^{\text {corr }}$. Both models make use of a multi-resolution time analysis, which is partially responsible for the accurate predictions in this listening condition by allowing the models to exploit the glimpses in the masker and thus capture the benefit in intelligibility. In the sEPSM $^{\text {corr }}$ framework, the correlation between the clean and processed internal representations is integrated across time windows using a "multiple-looks" integration (i.e., summing all squared correlations and taking the square root), which emphasizes the contributions of the higher modulation channels to SI in the framework of the sEPSM ${ }^{\text {corr }}$ since, as in SCASP, the number of the time windows is proportional to the modulation frequency. In contrast, in the sCASP model, the correlations obtained for each time window are averaged across time. Summation of correlation values is not necessary in sCASP, since its preprocessing includes an adaptation stage that compresses stationary signals to a higher degree than fast fluctuations, effectively acting as an envelope high-pass filter and thus emphasizing the contribution of the higher modulation bands in the internal representation. Therefore, the pre-processing of sCASP provides a weighting across modulation bands that is similar to the one provided by the backend of the sEPSM $^{\text {corr }}$. Both approaches suggest that higher-frequency modulation contributions evaluated in short time windows are important to account for SI in conditions with fluctuating interferers where speech information might be retrieved in the dips of the interferers.

The proposed sCASP model performs a crosscorrelation of the modulation-spectrograms of the template and target in its backend by means of an analysis of the relationship across frequency bands including both the time and audio-frequency axes. This approach is similar to the ones proposed in Carney (2018) and Scheidiger et al. (2018). Previously, Chabot-Leclerc et al. (2014) argued that in the condition of phase-jitter distortion the data could only be accounted for by the sEPSM framework when adding an explicit analysis across audio-frequencies, inspired by the work of Elhilali et al. (2003). Preliminary versions of the sCASP model that did not include this feature provided less accurate SI predictions for phase-jittered speech (cf. RelañoIborra et al., 2018). The sEPSM ${ }^{\text {corr }}$ model, which does not include an across-frequency analysis, did not correctly describe the (complete) loss of intelligibility observed in the listeners' data in some of the conditions. The improved performance of the sCASP model obtained by including the across-frequency analysis thus seems to support the findings of previous studies that argued for a spectro-temporal analysis of the speech signals to successfully assess SI.

Furthermore, the level analysis showed that sCASP can account for the effects of stimulation level on SI, thus reflecting processing limitations of the system both at low presentation levels (reflecting audibility limitations) and at high presentation levels (reflecting loss of frequency resolution and increased upwards spread of masking). Similar realistic SI predictions at different presentation levels were shown by the models of Scheidiger et al. (2018) and Zilany and Bruce (2007). Both approaches included non-linear stages in their auditory preprocessing, indicating that the active mechanisms reflected in the sCASP framework might be responsible for its level-dependent behavior. This is 
further supported by the inability of the linear EPSM $^{\text {corr }}$ to account for level effects.

\section{B. Limitations of the SCASP model}

The sCASP model showed a reduced accuracy in the condition of noisy speech enhanced with spectral subtraction, as described in Sec. IV C. This listening condition is peculiar in that, despite applying a noise-reduction algorithm, listeners reported higher SRTs (i.e., worse SI) with increasing amount of spectral subtraction $\kappa$ (Ludvigsen et al., 1990; Jørgensen and Dau, 2011). While sCASP correctly accounts for the trend of reduced SRTs with increased spectral subtraction factor, the model cannot capture the initial increase in SRT from the unprocessed condition to the $\kappa=0.5$ condition. This might be a direct consequence of the non-linear nature of the sCASP preprocessing, since employing noise reduction on the target input results in a target that will be processed more similarly to the template than in the case of the target in noise, yielding higher correlation values and hence lower SRT values. As a consequence, the model predicts improved intelligibility for the processed speech as compared to the unprocessed condition, which is not consistent with the data. Although similar effects might have been expected for the IBM processed-speech condition, since this algorithm also de-noises the input mixture, the IBM data are accounted for accurately by sCASP. The reason for this is that IBM processing increases intelligibility, as opposed to spectral subtraction where SI is reduced, thus increased correlations correspond to actual SI improvements in the IBMprocessed speech conditions. Indeed, the sCASP predictions were slightly more accurate than those of the SEPSM $^{\text {corr }}$ (see Table II). Additionally, the fitting condition for the Dantale II corpus that included all data points from the SSN interferer, including IBM processing, might have provided the model with sufficient information to robustly map SI to model outputs.

The fact that the target and template are processed differently due to the level-dependent non-linear nature of sCASP and the presence of, e.g., masker noises that affect the overall presentation level of the target, can result in undesired high correlation values. These effects might be overcome by using a model fitting condition that includes information about the specific processing in the target (instead of the speech in the presence of SSN only). However, this approach would reduce the generalizability of the model. Conditions with reverberant speech were not considered here, but they could pose a challenge to the model, as cross-correlation based models have consistently shown poor results in this condition (Relaño-Iborra et al., 2016). However, the performance in this condition could be improved if a reverberant template would be considered instead of the current dry and clean template. More generally, a principal limitation of the model is the need for a template itself. It implies that the model has a priori knowledge about the speech signals it receives, which is not necessarily the way speech perception occurs in humans. However, the proposed approach still allows for a thorough analysis of the speech cues that may be available in the different speech degradations, and their importance for SI.

\section{Perspectives}

The sCASP model could be valuable as a framework for predicting SI in HI listeners and subsequently to evaluate the efficacy of hearing-aid processing with regards to SI. The preprocessing originates from the CASP model, which was shown to successfully describe perceptual resolution limits related to human spectral, temporal and intensity processing both in NH and individual HI listeners (Jepsen et al., 2008; Jepsen and Dau, 2011). Therefore, a parametrization of the model's frontend to reproduce individual listeners' profiles is possible and has been shown to successfully predict basic psychoacoustic detection and masking data. The combination of the non-linear frontend and the short-time correlation analysis performed in the envelope domain seems to enable the sCASP model to capture relevant cues involved in SI prediction. An important next step will be to examine to what extent the model can account for the consequences of individual hearing loss on SI.

\section{CONCLUSION}

A new SI prediction model was presented, termed sCASP. The model represents an extension of the original CASP model (Jepsen et al., 2008) towards SI prediction. Several experimental conditions where speech was either degraded (due to background noise or phase-jitter distortions) or enhanced (by applying certain noise-reduction algorithms) were considered to evaluate the model's predictive power. Furthermore, the nonlinear behavior of the model was tested with regard to stimulation-level induced changes in NH SI. Based on the results obtained for $\mathrm{NH}$ listeners and the ability of the model frontend to account for perceptual resolution limits in basic psychophysical tasks in individual HI listeners, the sCASP model may provide a valuable framework to study the consequences of different types of sensorineural hearing loss on SI.

A MATLAB implementation of the sCASP model is available and may be requested from the authors via electronic mail.

\section{ACKNOWLEDGMENTS}

The authors would like to thank two anonymous reviewers for their very helpful and insightful comments. This work was supported by the Oticon Centre of Excellence for Hearing and Speech Sciences.

\section{APPENDIX}

The internal representation generated by the sCASP preprocessing can be understood as a three-dimensional (3D) matrix with audio frequency, modulation frequency, and time dimensions, represented by indices $i, j$, and $n$, respectively, in the internal representation matrix denoted $\boldsymbol{X}[i, j, n]$. The first step in the backend is to build the "modulation-based spectrograms," for each of the modulation channels. Let $j$ be the index of the selected modulation channel, then the number of selected audio bands for each modulation channel follows:

$$
I(j)=\sum_{i=1}^{m}\left[f_{c}(i)>4 \cdot f_{c, \text { mod }}(j)\right],
$$


where $f_{c}$ denotes the center frequency of an auditory channel and $f_{c, \text { mod }}$ the center frequency of a modulation channel, while $m$ represents the total number of DRNL channels, and $i$ represents the $i$ th auditory band. Here $[. .$.$] represent the$ Iverson brackets, defined to be 1 when the logical operation within them is true.

Once the range of the audio frequency axis has been defined for each modulation frequency independently, the modulation-spectrograms for each $j$ th modulation channel can be extracted from the internal representation as

$$
\boldsymbol{X}_{j}=\boldsymbol{X}\left(\begin{array}{ccc}
x_{i 1, j, n 1} & \cdots & x_{i 1, j, N} \\
\vdots & \ddots & \vdots \\
x_{I(j), j, n 1} & \cdots & x_{I(j), j, N}
\end{array}\right)
$$

where $\boldsymbol{X}$ is the original 3D internal representation and $\boldsymbol{X}_{j}$ is the $2 \mathrm{D}$ matrix, representing the modulation spectrogram for the $j$ th modulation frequency, $x_{j, i, n}$ indicates a unique element of the internal-representation $3 \mathrm{D}$ matrix for a certain audio frequency $(i)$, modulation frequency $(j)$, and time sample $(n)$. Thus, $\left(n_{1}, n_{2}, \ldots, N\right)$ represent the indices of the temporal samples vector, with $N$ being the sentence duration in samples, and $\left(i_{1}, i_{2}, \ldots, I(j)\right)$ represents the indices of the auditory channels considered for each modulation frequency, with $I(j)$ denoting the total number of selected audio channels for the $j$ th modulation channel, as previously defined.

Let $N_{s t}(j)=\left[1 / f_{c, \text { mod }}(j)\right] \cdot f_{s}$ be the number of time samples included in the short-term window of analysis for each modulation frequency (with $f_{s}$ indicating the sampling frequency). Then it follows that the short-term sections of the modulation-dependent spectrograms, noted $\boldsymbol{X}_{j, k}$ are defined as

$$
\boldsymbol{X}_{j, k}=\boldsymbol{X}\left(\begin{array}{ccc}
x_{i 1, j, n 1+k N_{\mathrm{st}}(j)} & \cdots & x_{i 1, j, N_{\mathrm{st}}(j)(1+k)} \\
\vdots & \ddots & \vdots \\
x_{I(j), j, n 1+k N_{\mathrm{st}}(j)} & \cdots & x_{I(j), j, N_{\mathrm{st}}(j)(1+k)}
\end{array}\right)
$$

where $k \in 0,1, \ldots, K(j)$, represents the indices of the temporal windows, with the total number of windows defined as $K(j)=\left\lfloor T / N_{\text {st }}(j)\right\rfloor$, where $T$ denotes the length of the speech signal in samples and $\lfloor\ldots\rfloor$ indicates the closest integer not greater to the expression within them. Using this short-time 2D segmentation the correlation metric for each time window and modulation subband is calculated as

$$
\chi_{j, k}=\frac{\left(\operatorname{vec}\left(X_{j, k}\right)-\operatorname{vec}\left(\bar{X}_{j, k}\right)\right) \cdot\left(\operatorname{vec}\left(\boldsymbol{Y}_{j, k}\right)-\operatorname{vec}\left(\overline{\boldsymbol{Y}}_{j, k}\right)\right)}{\left\|\operatorname{vec}\left(\boldsymbol{X}_{j, k}\right)-\operatorname{vec}\left(\bar{X}_{j, k}\right)\right\| \cdot\left\|\operatorname{vec}\left(\boldsymbol{Y}_{j, k}\right)-\operatorname{vec}\left(\overline{\boldsymbol{Y}}_{j, k}\right)\right\|},
$$

where $\operatorname{vec}\left(\boldsymbol{X}_{j, k}\right)$ is the vectorized version of the $2 \mathrm{D}$ modulation-spectrogram for the template (i.e., a onedimensional version of the matrix obtained by stacking all rows into one single vector), analogously $\operatorname{vec}\left(\boldsymbol{Y}_{j, k}\right)$ represents the vectorized version of the $2 \mathrm{D}$ modulation-spectrogram of the target, while $\overline{\boldsymbol{X}}$ and $\overline{\boldsymbol{Y}}$ represent the means of the modulationspectrograms of the template and the target, respectively. The metric is then averaged across time windows to obtain a correlation value per modulation frequency, $\chi_{j}$, by

$$
\chi_{j}=\frac{1}{K(j)} \cdot \sum_{k} \chi_{j, k}
$$

Finally, to obtain a single output value, the vector $\chi_{j}$ is averaged across modulation channels using

$$
\chi=\frac{1}{J} \cdot \sum_{j} \chi_{j}
$$

where $J$ is the total number of modulation channels in the internal representation and $\chi$ is the model's final output for each pair of target and template combination.

${ }^{1}$ Further analysis of the audibility and distortion components of the Plomp model is beyond the scope of this study.

${ }^{2}$ Human SRTs are only provided as a reference and should not be compared in absolute terms to the model predictions, as they were obtained using different speech materials and interferers.

Allen, J. B. (1996). "Harvey Fletcher's role in the creation of communication acoustics," J. Acoust. Soc. Am. 99(4), 1825-1839.

ANSI (1969). ANSI S3.5, "Methods for calculation of the articulation index" (American National Standards Institute, New York).

ANSI (1997). ANSI S3.5, "Methods for calculation of the speech intelligibility index" (American National Standards Institute, New York).

Berouti, M., Schwartz, R., and Makhoul, J. (1979). "Enhancement of speech corrupted by acoustic noise," in Proceedings of ICASSP '79, IEEE International Conference on Acoustics, Speech, and Signal Processing, Washington, DC, 1979, pp. 208-211.

Carney, L. H. (1993). "A model for the responses of low-frequency auditory-nerve fibers in cat," J. Acoust. Soc. Am. 93(1), 401-417.

Carney, L. H. (2018). "Supra-threshold hearing and fluctuation profiles: Implications for sensorineural and hidden hearing loss," J. Assoc. Res. Otolaryngol. 19(4), 331-352.

Chabot-Leclerc, A., Jørgensen, S., and Dau, T. (2014). "The role of auditory spectro-temporal modulation filtering and the decision metric for speech intelligibility prediction," J. Acoust. Soc. Am. 135(6), 3502-3512.

Ching, T. Y., Dillon, H., and Byrne, D. (1998). "Speech recognition of hearing-impaired listeners: Predictions from audibility and the limited role of high-frequency amplification," J. Acoust. Soc. Am. 103(2), 1128-1140.

Dau, T. (1996). "Modeling auditory processing of amplitude modulation," Ph.D. thesis, University of Oldemburg, Oldemburg.

Dau, T., Kollmeier, B., and Kohlrausch, A. (1997a). "Modeling auditory processing of amplitude modulation. I. Detection and masking with narrow-band carriers," J. Acoust. Soc. Am. 102, 2892-2905.

Dau, T., Kollmeier, B., and Kohlrausch, A. (1997b). "Modeling auditory processing of amplitude modulation. II. Spectral and temporal integration," J. Acoust. Soc. Am. 102, 2906-2919.

Dau, T., Püschel, D., and Kohlrausch, A. (1996). "A quantitative model of the 'effective' signal processing in the auditory system. I. Model structure," J. Acoust. Soc. Am. 99(6), 3615-3622.

Egan, J. P., and Hake, H. W. (1950). "On the masking pattern of a simple auditory stimulus,” J. Acoust. Soc. Am. 22(5), 622-630.

Elhilali, M., Chi, T., and Shamma, S. A. (2003). "A spectro-temporal modulation index (STMI) for assessment of speech intelligibility," Speech Commun. 41, 331-348.

Festen, J. M. (1993). "Contributions of comodulation masking release and temporal resolution to the speech-reception threshold masked by an interfering voice," J. Acoust. Soc. Am. 94(3), 1295-1300.

French, N. R., and Steinberg, J. C. (1947). "Factors governing the intelligibility of speech sounds," J. Acoust. Soc. Am. 19(1), 90-119.

Glasberg, B. R., and Moore, B. C. J. (1989). "Psychoacoustic abilities of subjects with unilateral and bilateral cochlear hearing impairments and their relationship to the ability to understand speech," Scand. Audiol. Suppl. 32, 1-25.

Glasberg, B. R., and Moore, B. C. J. (2000). "Frequency selectivity as a function of level and frequency measured with uniformly exciting notched noise," J. Acoust. Soc. Am. 108(5), 2318-2328.

Goode, R. L., Killion, M., Nakamura, K., and Nishihara, S. (1994). "New knowledge about the function of the human middle ear: Development of an improved analog model," Otol. Neurotol. 15(2), 145-154. 
Holube, I., Fredelake, S., Vlaming, M. S. M. G., and Kollmeier, B. (2010). "Development and analysis of an International Speech Test Signal (ISTS)," Int. J. Audiol. 49, 891-903.

Hornsby, B. W. Y., Trine, T. D., and Ohde, R. N. (2005). "The effects of high presentation levels on consonant feature transmission," J. Acoust. Soc. Am. 118(3), 1719-1729.

IEC (2003). IEC 60268-16:2003, "Sound system equipment-Part 16: Objective rating of speech intelligibility by speech transmission index" (International Electrotechnical Commision, Geneva, Switzerland).

ISO (1998). ISO 8253-1, "Audiometric test methods-Part 1: Pure-tone air and bone conduction audiometry" (International Organization for Standardization, Geneva, Switzerland).

ISO (2005). ISO 389-7, "Reference zero for the calibration of audiometric equipment-Part 7: Reference threshold of hearing under free-field and diffuse-field listening conditions" (International Organization for Standardization, Geneva, Switzerland).

Jepsen, M. L., and Dau, T. (2011). "Characterizing auditory processing and perception in individual listeners with sensorineural hearing loss," J. Acoust. Soc. Am. 129(1), 262-281.

Jepsen, M. L., Ewert, S. D., and Dau, T. (2008). “A computational model of human auditory signal processing and perception,” J. Acoust. Soc. Am. 124(1), 422-438.

Jørgensen, S., and Dau, T. (2011). "Predicting speech intelligibility based on the signal-to-noise envelope power ratio after modulation-frequency selective processing," J. Acoust. Soc. Am. 130(3), 1475-1487.

Jørgensen, S., Ewert, S. D., and Dau, T. (2013). "A multi-resolution envelope-power based model for speech intelligibility," J. Acoust. Soc. Am. 134(1), 436-446.

Kjems, U., Boldt, J. B., Pedersen, M. S., Lunner, T., and Wang, D. (2009). "Role of mask pattern in intelligibility of ideal binary-masked noisy speech,” J. Acoust. Soc. Am. 126(3), 1415-1426.

Kollmeier, B., Schädler, M. R., Warzybok, A., Meyer, B. T., and Brand, T. (2016). "Sentence recognition prediction for hearing-impaired listeners in stationary and fluctuation noise with FADE: Empowering the attenuation and distortion concept by Plomp with a quantitative processing model," Trends Hear. 20, 1-17.

Langner, G., and Schreiner, C. E. (1988). "Periodicity coding in the inferior colliculus of the cat. I. Neuronal mechanisms," J. Neurophysiol. 60(6), $1799-1822$.

Lopez-Poveda, E. A. (2014). "Why do I hear but not understand? Stochastic undersampling as a model of degraded neural encoding of speech," Front. Neurosci. 8, 348.

Lopez-Poveda, E. A., and Meddis, R. (2001). "A human nonlinear cochlear filterbank," J. Acoust. Soc. Am. 110(6), 3107-3118.

Lorenzi, C., Gilbert, G., Carn, H., Garnier, S., and Moore, B. C. J. (2006). "Speech perception problems of the hearing impaired reflect inability to use temporal fine structure," Proc. Natl. Acad. Sci. U.S.A. 103(49), 18866-18869.

Ludvigsen, C., Elberling, C., Keidser, G., and Poulsen, T. (1990). "Prediction of intelligibility of non-linearly processed speech," Acta OtoLaryngol. Suppl. 469, 190-195.

Meyer, R. M., and Brand, T. (2013). "Comparison of different short-term speech intelligibility index procedures in fluctuating noise for listeners with normal and impaired hearing," Acta Acust. united Acust. 99(3), 442-456.

Nielsen, J. B., and Dau, T. (2009). "Development of a Danish speech intelligibility test," Int. J. Audiol. 48, 729-741.

Nilsson, M., Soli, S. D., and Sullivan, J. A. (1994). "Development of the Hearing In Noise Test for the measurement of speech reception thresholds in quiet and in noise," J. Acoust. Soc. Am. 95(2), 1085-1099.

Pavlovic, C. V. (1987). "Derivation of primary parameters and procedures for use in speech intelligibility predictions," J. Acoust. Soc. Am. 82(2), 413-422.

Pavlovic, C. V., Studebaker, G. A., and Sherbecoe, R. L. (1986). "An articulation index based procedure for predicting the speech recognition performance of hearing-impaired individuals," J. Acoust. Soc. Am. 80(1), $50-57$.

Payton, K. L., and Uchanski, R. M. (1994). "Intelligibility of conversational and clear speech in noise and reverberation for listeners with normal and impaired hearing," J. Acoust. Soc. Am. 95, 1581-1592.

Peters, R. W., Moore, B. C. J., and Baer, T. (1998). "Speech reception thresholds in noise with and without spectral and temporal dips for hearing-impaired and normally hearing people," J. Acoust. Soc. Am. 103(1), 577-587.
Pickett, J. M., and Pollack, I. (1958). "Prediction of speech intelligibility at high noise levels," J. Acoust. Soc. Am. 30(10), 955-963.

Plomp, R. (1978). "Auditory handicap of hearing impairment and the limited benefit of hearing aids," J. Acoust. Soc. Am. 63(2), 533-549.

Plomp, R. (1986). "A signal-to-noise ratio model for the speech-reception threshold of the hearing impaired," J. Speech Hear. Res. 29(2), 146-154.

Pralong, D., and Carlile, S. (1996). "The role of individualized headphone calibration for the generation of high fidelity virtual auditory space," J. Acoust. Soc. Am. 100(6), 3785-3793.

Püschel, D. (1988). "Prinzipien der zeitlichen Analyse beim Hören" ("Principles of temporal analysis in the auditory system"), University of Göttingen.

Relaño-Iborra, H., May, T., Zaar, J., Scheidiger, C., and Dau, T. (2016). "Predicting speech intelligibility based on a correlation metric in the envelope power spectrum domain," J. Acoust. Soc. Am. 140(4), 2670-2679.

Relaño-Iborra, H., Zaar, J., and Dau, T. (2018). "Extending a computational model of auditory processing towards speech intelligibility prediction," in Proceedings of the International Symposium on Auditory and Audiological Research, 23-25 August 2017, Nyborg, Denmark, pp. 319-326.

Rhebergen, K. S., Versfeld, N. J., De Laat, J. A. P. M., and Dreschler, W. A. (2010). "Modelling the speech reception threshold in non-stationary noise in hearing-impaired listeners as a function of level," Int. J. Audiol. 49(11), 856-865.

Schädler, M. R., Hülsmeier, D., Warzybok, A., Hochmuth, S., and Kollmeier, B. (2016). "Microscopic multilingual Matrix test predictions using an ASRbased speech recognition model," in Proceedings of the Annual Conference of the International Speech Communication Association, INTERSPEECH, September 8-12, San Francisco, CA, pp. 610-614.

Schädler, M. R., Warzybok, A., Hochmuth, S., and Kollmeier, B. (2015). "Matrix sentence intelligibility prediction using an automatic speech recognition system," Int. J. Audiol. 54, 100-107.

Scheidiger, C., Carney, L. H., Dau, T., and Zaar, J. (2018). "Predicting speech intelligibility based on across-frequency contrast in simulated auditorynerve fluctuations," Acta Acust. united Acust. 104(5), 914-917.

Speaks, C., Karmen, J. L., and Benitez, L. (1967). "Effect of a competing message on synthetic sentence identification," J. Speech Hear. Res. 10(2), 390-395.

Steeneken, H. J. M., and Houtgast, T. (1980). "A physical method for measuring speech-transmission quality," J. Acoust. Soc. Am. 67(1), $318-326$.

Strelcyk, O., and Dau, T. (2009). "Relations between frequency selectivity, temporal fine-structure processing, and speech reception in impaired hearing," J. Acoust. Soc. Am. 125(5), 3328-3345.

Studebaker, G. A., Sherbecoe, R. L., McDaniel, D. M., and Gwaltney, C. A. (1999). "Monosyllabic word recognition at higher-than-normal speech and noise levels," J. Acoust. Soc. Am. 105(4), 2431-2444.

Summers, V., and Cord, M. T. (2007). "Intelligibility of speech in noise at high presentation levels: Effects of hearing loss and frequency region," J. Acoust. Soc. Am. 122(2), 1130-1137.

Summers, V., and Molis, M. R. (2004). "Speech recognition in fluctuating and continuous maskers," J. Speech Lang. Hear. Res. 47(2), 245.

Taal, C. H., Hendriks, R. C., Heusdens, R., and Jensen, J. (2011). "An algorithm for intelligibility prediction of time-frequency weighted noisy speech," IEEE Trans. Audio Speech Lang. Process. 19(7), 2125-2136.

van Wijngaarden, S. J., and Steeneken, H. J. M. (1999). "Objective prediction of speech intelligibility at high ambient noise levels using the speech transmission index," in Proceedings of the 6th European Conference on Speech Communication and Technology, 5-9 September 1999, Budapest, Hungary.

Verhey, J. L., Dau, T., and Kollmeier, B. (1999). "Within-channel cues in comodulation masking release (CMR): Experiments and model predictions using a modulation-filterbank model," J. Acoust. Soc. Am. 106(5), 2733-2745.

Wagener, K. C., Lignel Josvassen, J., and Ardenkjaer, R. (2003). "Design, optimization and evaluation of a Danish Sentence Test in Noise," Int. J. Audiol. 42(1), 10-17.

Zilany, M. S. A., and Bruce, I. C. (2007). "Predictions of speech intelligibility with a model of the normal and impaired auditory-periphery," Proceedings of the 3rd International IEEE EMBS Conference on Neural Engineering, May 2-5, Kohala Coast, HI, pp. 481-485.

Zilany, M. S. A., Bruce, I. C., and Carney, L. H. (2014). "Updated parameters and expanded simulation options for a model of the auditory periphery," J. Acoust. Soc. Am. 135(1), 283-286. 